\title{
Current status and prospects of androgen depletion therapy for
}

\section{prostate cancer}

Hideyuki Akaza, Department of Urology,

University of Tsukuba, Tsukuba, Ibaraki, Japan

akazah@md.tsukuba.ac.jp

Introduction

It is said that the prostate was histologically acknowledged as an independent organ for the first time in the mid-nineteenth century. No organ termed the prostate is indicated even in the world-renowned drawing of anatomy by Leonard da Vinci (1452 - 1519). It was in 1941 that androgen depletion therapy (ADT) was reported as effective against prostate cancer. For this achievement, Dr. Huggins received a Nobel Prize 25 years later in 1966. At that time, most patients with prostate cancer who received ADT were in progressive stage. As such, Dr. Huggins himself observed that recurrence was noted in many cases after several years of remission. ADT was therefore classified as a palliative treatment. Since PSA was discovered in 1979, the rate of detection of early-stage prostate cancer has rapidly improved. Subsequently, in 1983, the method of anatomical prostatectomy was reported by Walsh, and established the concept of radical treatment of prostate cancer. To be more precise, it became accepted that localized prostate cancer (LPC) and locally advanced prostate cancer (LAPC), the rate of detection of which sharply increased thanks to PSA screening, should be aggressively treated by radical methods including prostatectomy and radiotherapy. However, the conclusion that ADT is a palliative method of treatment reached half a century ago is still accepted at present. As a result, this concept has been applied even to LPC and LAPC, and few careful clinical studies have been performed ${ }^{1 \text { ). }}$ Despite the progress in ADT, including the establishment of theoretical grounds for maximal androgen blockade (or combined androgen blockade; $\mathrm{MAB}$ or $\mathrm{CAB}$ ) and the development of a new nonsteroidal antiandrogen preparation, there has been little change in the concept of ADT as a palliative method of treatment of progressive prostate cancer, either 
The Prostate Cancer Treatment Guideline was published by the Japanese Urological Association in $2006^{2)}$. As a member in charge of the drug therapy section of this edition, the author would like to report the current status of ADT by citing the abstract of the overview of this section.

“At present, there is no chemotherapy superior to endocrine therapy for the treatment of prostate cancer. Though the proximal effects of various endocrine therapies are remarkable, there is a limit to their indication since the effects of treatment persist for only for 2 to 3 years in progressive cases and because of the onset of sex-related adverse reactions including erectile dysfunction (ED) and decreased libido.

The most generally applicable endocrine therapy is monotherapy with luteinizing hormone-releasing hormone (LH-RH) agonist or antiandrogen preparation, or concomitant treatment with these 2 drugs. In treatment with LH-RH agonist, concomitant administration of an antiandrogen preparation should be considered if urinary tract obstruction caused by flare-up phenomenon associated with a transient increase in testosterone in the initial stage of administration, ostealgia due to a metastatic lesion, spinal compression, etc. are anticipated. The efficacy of LH-RH agonist is considered equivalent to that of castration. However, the efficacy of monotherapy with antiandrogen preparation is reported to be less than that of LH-RH, though no significant difference was observed. On the other hand, antiandrogen preparations induce fewer sex-related adverse reactions. Monotherapy with nonsteroidal antiandrogen preparations has therefore been reported to be useful, depending on patient status. When the usefulness of bicalutamide as auxiliary therapy was investigated in LPC or LAPC in patients undergoing radical surgery, radiotherapy, and cautious observation of clinical course, prolongation of PSA doubling time and decrease in objective risk of progression were observed. At present, a large-scale investigation of prolongation of survival period is proceeding. In another investigation, whether chemo-endocrine therapy is more effective in treating Stage IV prostate cancer than endocrine monotherapy alone is being examined.

The down-staging effect of neo-adjuvant hormonal therapy (NHT) is manifested as a decrease in frequency of cases with a positive resected stump or lymph node metastasis. Many randomized controlled studies (RCT) have confirmed the efficacy of NHT. However, since no clear evidence that NHT can improve survival rate has been obtained, 
the results of analysis of long-term prognosis in each of these trials are awaited. Endocrine therapy is opted for in treating stage III-IV prostate cancer, and life-prolonging effects of it have been observed. Endocrine therapy is sometimes performed in combination with surgery and radiotherapy in stage III cases. Particularly since the report on the results of a RCT that survival period was prolonged more by concomitant radiotherapy and endocrine therapy than by radiotherapy alone, there has been much expectation regarding the efficacy of concomitant treatment. However, there are problems in this regard, including the selection of appropriate patients, the appropriate time for initiation of endocrine therapy, the duration of treatment, and others. In general, the standard treatment for progressive prostate cancer with metastasis is ADT by surgical or medical castration. Since $40 \%$ of the androgen present in prostate gland cells appears to be derived from the adrenals, MAB was found to be useful due to inhibition of androgen from the testes and adrenals via castration (orchiectomy or LH-RH agonist administration) in combination with nonsteroidal antiandrogen preparation administration. Though MAB has been a generally accepted method of treatment of progressive prostate cancer, whether it can improve long-term prognosis in comparison with castration alone has been a subject of recent discussion. According to the results of a meta-analysis recently performed, there is little difference in 2-year survival rate between MAB and monotherapy (castration or LH-RH agonist alone), though 5-year survival rate was significantly higher with MAB. However, since the difference in survival rate is small, it appears that true clinical benefit should be determined considering adverse reactions, QOL, and cost. At present, the results of further research demonstrating the validity of MAB are awaited. Recently, the results of a double-blind study that compared MAB monotherapy using bicalutamide as antiandrogen preparation with $\mathrm{LH}-\mathrm{RH}$ agonist monotherapy were reported. Findings indicated a definite effect on time to progression. Even when recurrence of cancer is observed, discontinuation of antiandrogen preparation alone can in some cases transiently inhibit the progress of disease (antiandrogen withdrawal syndrome). PSA decreased in $14-60 \%$ and a clinical effect was observed in 0 - 25\% of patients with discontinuation of antiandrogen preparation, or combination with hydrocortisone. However, PSA reduction is reported to persist for only for 2 to 4 months, in general.

The above findings do not differ notably from those noted in the guidelines of the EAU or 
NCI-PDQ. However, the EAU and NCI-PDQ guidelines take a somewhat negative stance regarding use of ADT in the treatment of LPC and LAPC.

The current issues in use of ADT concern the following 3 points: 1) Though their number is still small, clinical studies based on new concepts (effects of MAB and intermittent androgen blockade therapy, etc.) are being performed. Depending on the results of these studies, new conclusions regarding the role of ADT may be reached. 2) ADT is generally employed in Japan to treat LPC and LAPC (Fig. 1), and MAB is used in many cases (Fig. 2). This tendency is definitely on the increase in the United States, as well (Fig. 3). 3) This choice of treatment is inconceivable if treatment method is selected according to the guidelines. Why, then, does it exist? 
Fig. 1

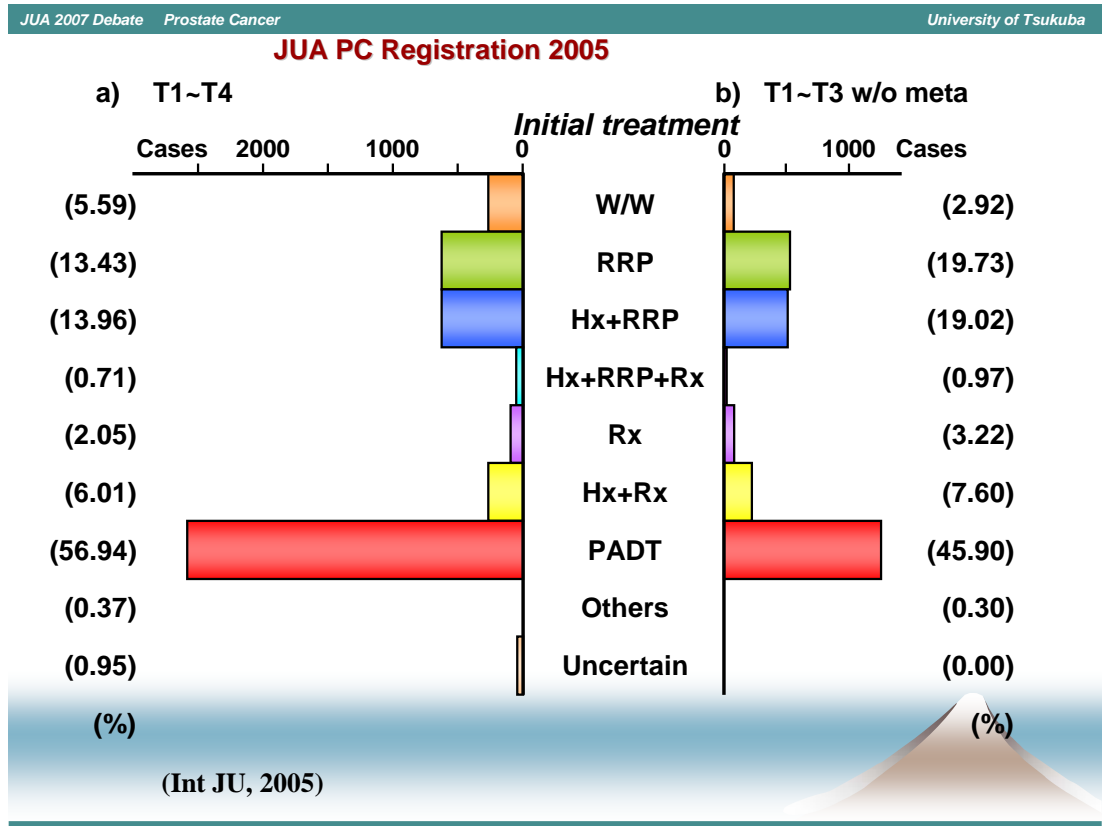

Prostate cancer registration data provided by the Japanese Urological Association are shown in this graph.

Types of initial treatment as of 2000 for newly diagnosed patients are summarized.

PADT was selected for 56.94\% of cases of T1 through T4 disease. For T1 through T3, PADT was still selected for $45.90 \%$ of cases.

Cancer Registration Committee of the Japanese Urological Association.

Clinicopathological statistics on registered prostate cancer patients in Japan: 2000 Report from the Japanese Urological Association.

Int J Urol. 2005;12:46-61. 
Fig. 2

JUA 2007 Debate Prostate Cancer

\section{Method of PADT in Japan \\ J CaP surveillance}

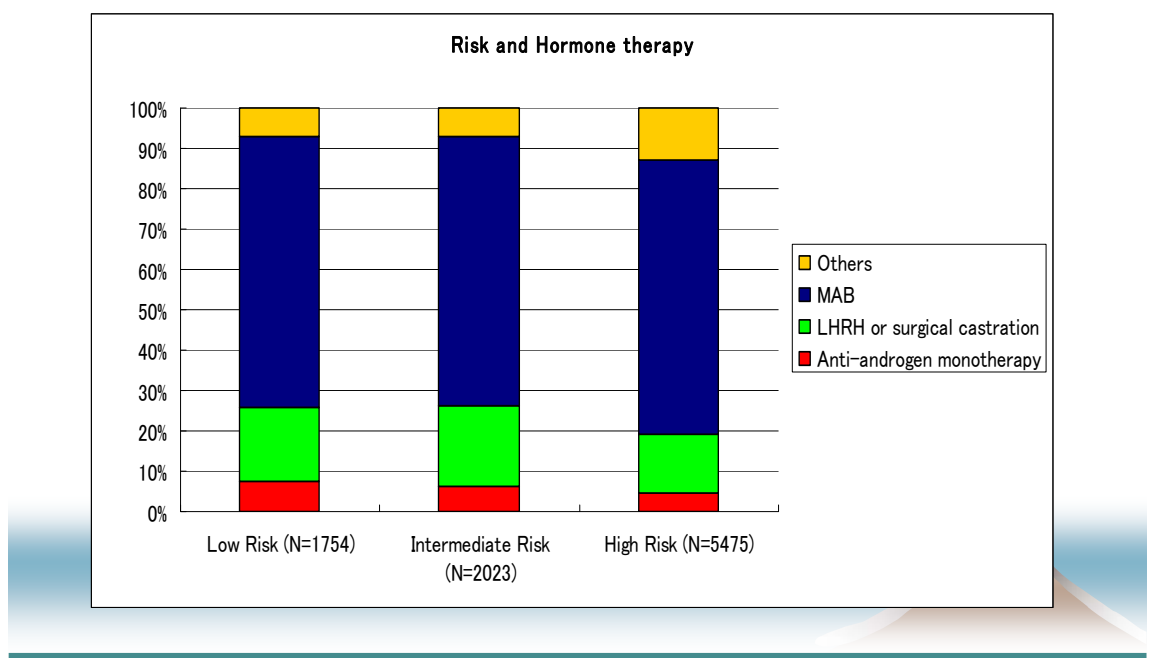

Results of survey conducted by the Japan Prostate Cancer Study Group (JCaP)

The results indicate that MAB is used as PADT in general in both low-risk and high-risk groups.

Akaza H, Hinotsu S, Usami M, et al. Characteristics of patients with prostate cancer who have initially been treated by hormone therapy in Japan: J-CaP surveillance.

Jpn J Clin Oncol. 2004;34:329-36. 
Fig. 3

\section{Trend of PADT for localized prostate cancer in U.S.A.}

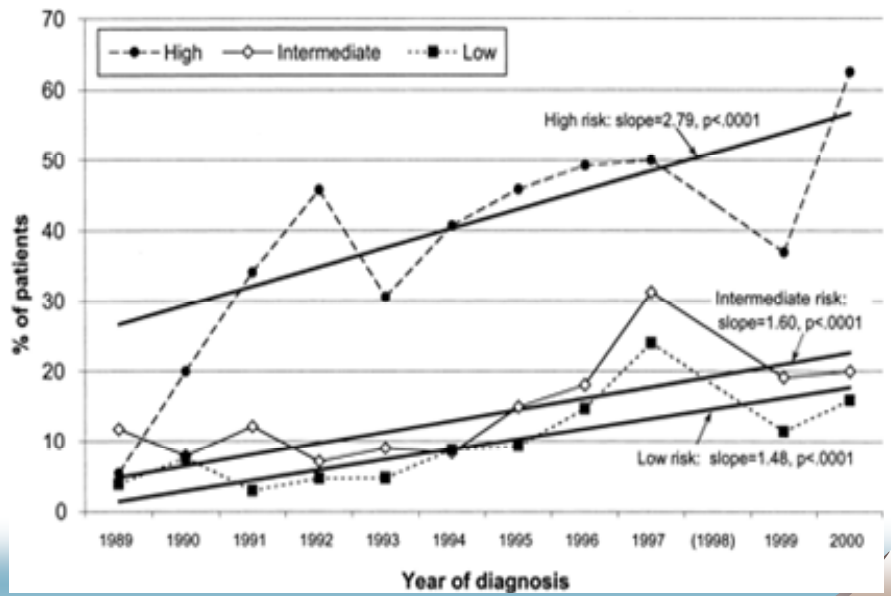

(Cooperberg, et al. JNCl 2003)

\section{USA CaPSURE data}

PADT is increasingly selected each year as a treatment against localized prostate cancer including the low risk and high risk groups. However, the ratio is still lower in comparison with the PADT ratio in Japan (Fig. 1).

Cooperberg MR, Grossfeld GD, Lubeck DF, Carroll PR. National practice patterns and time trends in androgen ablation for localized prostate cancer.

J Natl Cancer Inst. 2003;95:981-9. 
(III) Factors involved in the determination of method of treatment According to Mulrow ${ }^{3)}$, there are 3 types of factors that the physician and patient take into consideration in determining method of treatment (Fig. 4): (A) evidence, (B) patient/physician factors, and (C) constraints. Referring to Fig. 4, guidelines are established based on A and C, while B and C are related to ethical aspects of treatment, and $\mathrm{A}$ and $\mathrm{B}$ involve knowledge. Thus, the guidelines alone are not sufficient for determination of treatment in general clinical practice. Ethical issues, education, experience, and personal sense of value and cultural factors are also involved in decisions regarding treatment. In regard to the selection of endocrine therapy, there is less evidence, since only a few careful clinical studies have been performed. If evidence is insufficient, neither affirmative nor negative judgments are possible regarding treatment methods generally used at present. However, if this is the case, why is a negative stance taken in Europe and the United States regarding prostate androgen blockage monotherapy (PADT), and why is PADT increasingly performed at present despite this? Why is PADT accepted more and why is MAB mostly employed in Japan?

As shown in Fig. 4, elements B and C differ among countries, which has complex effects on the situation. Fleming et al. ${ }^{4)}$ examined the relationships of prostatectomy, external radiotherapy, and observation of clinical course (slow ADT) to prognosis (death or onset of metastasis) of patients on the basis of the literature and medicare claims data. Though surgery and radiotherapy are effective in young patients, there is not much benefit to them, and the benefit obtains varies among patients. In fact, it has been concluded that the observation of clinical course (slow ADT) is a valid choice for patients over 70 years of age. An astute observer may interpret this report as a warning against unnecessary surgery and radiotherapy under the influence of various requirements. Similar findings have been reported by Miller et al. ${ }^{5}$. 
Fig. 4

\section{Factors that enter into clinical decisions}

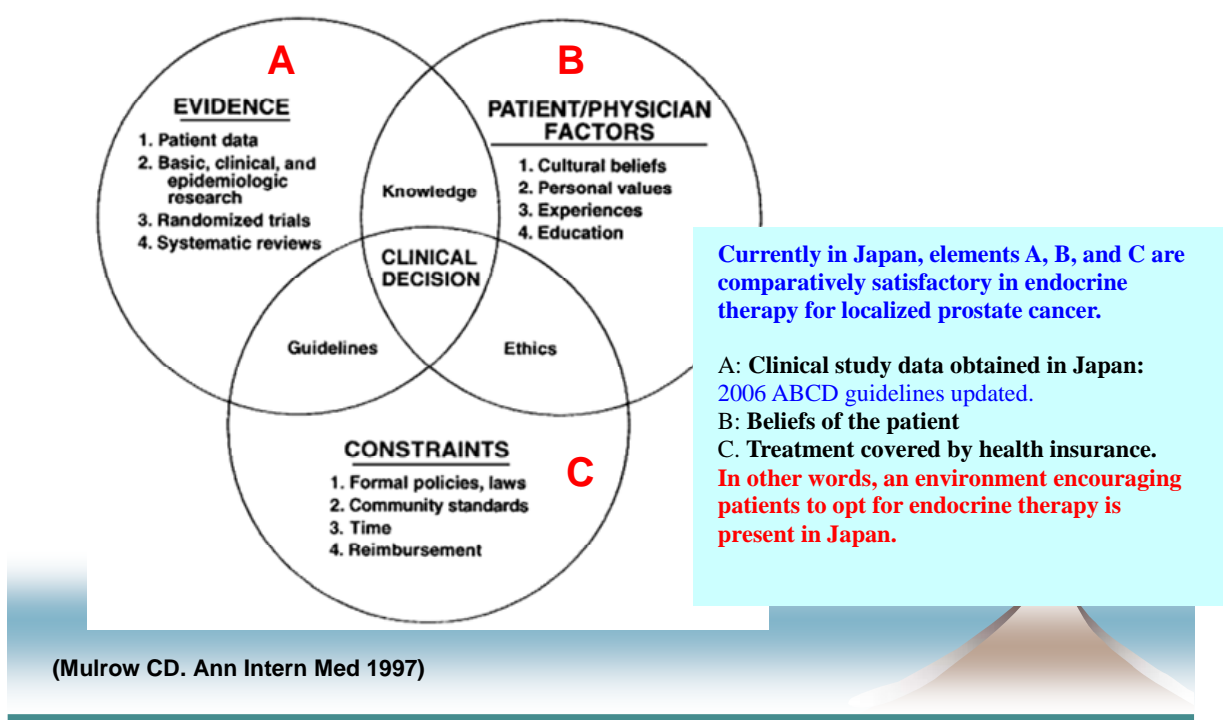

Three elements related to treatment

Mulrow C, Langhome P, Grimshaw J. Integrating heterogeneous pieces of evidence in systematic reviews.

Ann Intern Med. 1997;127:989-95. 
(VI) Present-day ADT based on the Update of the 2006 ASCO Guidelines In April 2007, the 2004 edition of the ASCO treatment guidelines for PADT in recurrent or progressive prostate cancer with metastasis was updated ${ }^{6)}$. In these guidelines, the following 5 issues are discussed on the basis of newly published clinical study results: (1) What are the standard initial treatment options? (2) Are antiandrogens as effective as other castration therapies? (3) Is combined androgen blockade better than castration alone? (4) Does early androgen-deprivation therapy improve outcome compared with deferred therapy? (5) Is intermittent androgen deprivation therapy better than continuous androgen deprivation therapy? The essence of ADT is captured in each of these 5 issues. These issues are in addition important in assessing the usefulness of ADT in LPC and LAPC, the issue of greatest importance to use of ADT at present. The amount of literature judged to contribute to this update is very small during the relevant period, and is limited to 12 reports including those on RCT and reviews. Concerning issues (1) and (2), in particular, no new study results have been published (accepted). Therefore, no updating has been performed regarding these 2 issues. On the other hand, the guidelines have been revised to clearly recommend "consideration of CAB using bicalutamide" on the basis of one RCT and one analytical research study. Concerning issue (4), the best time to start PADT, detailed analysis has been on the basis of 12 reports including 10 RCTs. Though the risk of death by cancer was reduced by $17 \%$ in patients with metastasis or progressive patients when ADT was started earlier, overall survival rate was not prolonged. For recurrent prostate cancer, the guidelines recommend performance of clinical studies if possible. Regarding issue (5), intermittent ADT, updating was performed on the basis of results of one RCT. However, it has been concluded that the data are insufficient at present to recommend intermittent ADT in clinical practice. Large-scale clinical studies currently in progress are introduced in this ASCO update. They include 2 large-scale studies related to the time of initiation of ADT and 2 other large-scale studies related to intermittent ADT, which also intend to investigate QOL. The results of these studies are expected to demonstrate the role of ADT more clearly. 
(V) Conservative management: Ambiguous interpretation as watchful waiting or active surveillance

Recently, the concepts of watchful waiting and active surveillance have become subjects of discussion, and have (as so-called conservative management) been included among the choices recommended for patients with low-risk prostate cancer and elderly patients.

The findings of large retrospective studies have provided the basis for this concept. However, reading of reports appearing in world-renowned medical reviews makes it clear that there is no clear distinction between conservative management and PADT for LPC, and that the description in this regard is ambiguous.

According to the famous report by Albertesen et al. entitled "20-year outcomes following

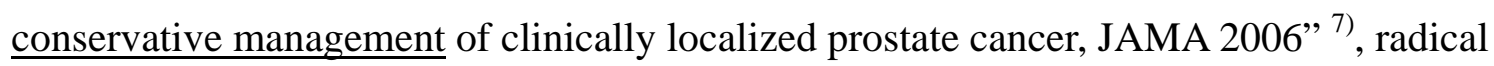
treatment is not necessary and conservative management is sufficient for low-risk or elderly patients. However, in their study $42 \%$ of patients received endocrine therapy within 6 months after diagnosis. According to the report by Miller et al. (JNCI2006) 8), the initial treatment method in LPC was classified by age at time of diagnosis and biopsy grade, though appropriate treatment, that is, expectant management or PADT, was selected for only $45 \%$ of the low-risk group patients while the majority underwent prostatectomy and radiotherapy, which are considered excessive treatment. The influence of reports appearing in well-read and famous medical reviews on clinical practice is not to be overlooked. This report should be interpreted as handling PADT as an aggressive method of treatment with the outcome being influenced by PADT rather than regarding the report simply as an outcome of conservative management. This suggests the need for a randomized study of treatment of LPC and LAPC assigning subjects to immediate ADT and delayed ADT. The author considers it unwise to too readily recommend watchful waiting or conservative management until the results of further study have been validly assessed. 
(VI) Future of ADT

A considerable number of malignant tumors are not localized to the organs of primary onset, and have already metastasized (clinically or microscopically) at the time of diagnosis. Furthermore, surgery sometimes fails to completely extirpate LPC and LAPC, resulting in subsequent local recurrence and remote metastasis. Systemic therapy is thus important in the treatment of cancer. In the case of prostate cancer, ADT is a powerful means of systemic treatment. Thanks to the success of a large-scale prostate cancer prevention trial (PCPT) ${ }^{8)}$ using the $5 \alpha$ reductase inhibitor finasteride, safe endocrine therapy that can maintain QOL has become possible. The establishment of more powerful methods of ADT without impairment of QOL is expected not only for patients with progressive prostate cancer but also for LPC and LAPC, through the development of new antiandrogen preparations and molecular target drugs.

It is important to promote basic and clinical research based on the understanding that cure of prostate cancer is almost always possible with current ADT if progression to the hormone independent prostate cancer can be avoided. 


\section{References}

1) Akaza $H$. Trends in primary androgen depletion therapy for patients with localized and locally advanced prostate cancer: Japanese perspective. Cancer Sci. 2006;97:243-7.

2) The Prostate Cancer Treatment Guideline 2006 edited by Japanese Urological Association. KANEHARA \& CO., LTD. 2006.

3) Mulrow C, Langhome P, Grimshaw J. Integrating heterogeneous pieces of evidence in systematic reviews.

Ann Intern Med. 1997;127:989-95.

4) Fleming $\mathrm{C}$, Wasson $\mathrm{JH}$, Albertsen $\mathrm{PC}$, et al. A decision analysis of alternative treatment strategies for clinically localized prostate cancer. Prostate Patient Outcomes Research Team. JAMA. 1993;269:2650-8.

5) Miller DC, Gruber SB, Hollenbeck BK, et al. Incidence of initial local therapy among men with lower-risk prostate cancer in the United States. J Natl Cancer Inst. 2006;98:1134-41.

6) Loblaw DA, Virgo KS, Nam R, et al. Initial hormonal management of androgen-sensitive metastatic, recurrent, or progressive prostate cancer: 2006 update of an american society of clinical oncology practice guideline.

J Clin Oncol. 2007;25:1596-605. Epub 2007 Apr 2.

7) Albertsen PC, Hanley JA, Fine J. 20-year outcomes following conservative management of clinically localized prostate cancer. JAMA. 2005;293:2095-101.

8) Ankerst DP, Thonpson IM. New answers from the Prostate Cancer Prevention Trial on the chemoprevention of prostate cancer. Arch Ital Urol Androl. 2006;78:154-6. 